\title{
Proceedings of the 17th Annual Meeting of the Society for Pediatric Dermatology, July 29-31, 1993, Snowmass, Colorado
}

Anne Lucky, M.D., ${ }^{*}$ Lawrence F. Eichenfield, M.D., $\dagger$ A. Howland Hartley, M.D., $\ddagger$ and James E. Rasmussen, M.D.§

¿Dermatology Clinic, Children's Hospital Medical Center, Cincinnati, Ohioj] tDivision of Pediatric Dermatology, Children's Hospital and University of California, San Diego School of Medicine, San Diego, California; $¥$ Section of Pediatric Dermatology, Children’s National Medical Center, Washington, D.C.; and §Department of Dermatology and Pediatrics, University of Michigan Medical Center, Ann Arbor, Michigan

Thursday, July 29, 1993

The first half of the morning session was devoted to genetics as related to dermatology.

Dr. Virginia Sybert (University of Washington) discussed the vocabulary of medical genetics. Since Mendel described the four basic modes of inheritance (autosomal dominant, autosomal recessive, $\mathrm{X}$-linked dominant, and $\mathrm{X}$-linked recessive) there has been an explosion of knowledge that is often inaccessible to those who do not understand the new vocabulary. Dr. Siebert described the basic gene structure of DNA, explaining the difference between exons, the expressing sequences, and introns, the intervening sequences. During transcription to RNA, the introns are cleaved out, and the exons code for the final product. Alleles are different versions of the same gene on the same locus of one chromosome. Polymorphisms represent variations in DNA sequence among individuals that occur with a frequency of at least $1 \%$ in the population. Restriction fragment length polymorphisms (RFLPs) use bacterial enzymes to cleave DNA into small pieces, which can then be compared. Linkage defines two gene loci in close proximity and represents genes that tend to be inherited together. Linkage analysis, a powerful tool used to define location of specific genes on the chromosome, is dependent on DNA polymorphisms or on allelic variations. Crossovers, exchanges of genetic material during mitosis, can confound linkage analysis. In general, DNA diagnoses can be made indirectly by linkage analysis or directly by DNA probes to obtain the specific gene sequence.

Dr. Siebert also described unusual modes of inheritance, including imprinting, in which maternal or paternal transmission affects expressivity of a disorder. This is seen in myotonic dystrophy, which is more severe in offspring of affected mothers than in offspring of affected fathers. Another unusual inheritance pattern is mitochondrial, which is only maternal. Contiguous gene syndromes, which in the past were thought to represent several effects of a single variation, are now believed to represent the effect of abnormalities in a group of closely linked genes. Finally, mosaicism has recently become recognized as an important mode of inheritance explaining many of the patterned dermatologic diseases that we see. Mosaicism, defined as the presence of two or more genetically or chromosomally distinct cell lines in one zygote, can be somatic and present only in tissue, can be gonadal and thus passed on, or both. Some segmental diseases often expressed in the lines of Blaschko, such as epidermal nevi and incontinentia pigmenti, may be only somatic or somatic and gonadal. Partial lethal mutations can result in severe deformities, such as in the CHILD syndrome. Finally, chromosomal mosiacism in which there are more than two chromosomal cell lines, has been found to cause some of the unusual disorders, such as hypomelanosis of Ito. Functional $X$ inactivation (or lyonization) accounts for variable expressivity, depending on which proportion of normal or abnormal X chromo- 
somes is inactivated. Dr. Siebert highly recommended the book Dealing With Genes: The Language of Heredity, by Paul Berg and Maxine Singer.

The second speaker was Dr. Ervin Epstein, Jr. (University of California at San Francisco), who discussed epidermolysis bullosa simplex and keratin disorders. He reviewed various strategies for diagnosing genetic diseases. The original pattern was to identify the disorder, such as albinism, look for a functional abnormality, such as melanogenesis, postulate the absence of an enzyme, such as tyrosinase, and then identify mutations in the enzyme and localize them to the chromosome, in this case, chromosome 11. An alternative method would be to use linkage analysis to identify which gene in a family with a particular disorder has a mutation and, after the gene is identified, try to identify its function.

In epidermolysis bullosa (EB), the disorders have been grouped because of their clinical appearance; however, it is now clear that recessive dystrophic EB represents an abnormality of the collagen of the anchoring fibrils, and EB simplex seems to be a keratin disorder in which defective intermediate filaments form a three-dimensional cytoskeleton structure of the keratinocyte. With abnormalities in the keratin structure, cells collapse, and the normal, orderly processes of keratinization are disrupted. Mutations in basal cell keratins 5 and 14 have been identified in a variety of families with EB simplex. Genes for these keratins are found specifically on the long arms of chromosomes 12 and 17. Dr. Epstein summarized the large number of cutaneous disorders that have been localized to specific chromosomes to date and shared a table from his recent publication (Table 1).
The third speaker, Dr. Dennis Rupe (Baylor University) described studies that he and colleagues, including Dr. Andrea Dominey, performed on material from patients with epidermolytic hyperkeratosis (EHK). As opposed to EB simplex, which affects the basal cells containing keratins 5 and 14, the abnormality in EHK is in the spinous layer involving keratins 1 and 10 . Keratins of types 1 and 2 must be able to achieve their ideal three-dimensional structure by forming a dimer, and a mutation that prevents this normal dimer formation, such as a deletion in one chain, could cause abnormalities of keratin. To date, a variety of point mutations at various positions on the gene coding $\mathrm{K} 1$ and $\mathrm{K} 10$ have been identified. Most of these appear to occur at the ends of the genes where filaments overlap. One hypothesis for the pathophysiology of the ichthyotic component of EHK is that the keratin mutations cause cytolysis, which subsequently releases cytokines, which cause failure of normal feedback to reduce basal cell proliferation.

Dr. Rupe talked about future prospects in molecular genetics as related to dermatology. Prenatal diagnosis requires knowledge of the specific mutation for each family. Once that is known, chorionic villus sampling could be done at 10 to 12 weeks of gestational age instead of at 20 to 24 weeks, when intrauterine skin biopsy is now commonly done. Finally, there is a potential for analyzing in vitrofertilized eggs with known risk for genetic diseases.

A second future prospect is gene therapy. Theoretically, gene therapy could be used to inhibit expression of a mutant allele by creating an antisense RNA or creating ribozymes that can cause degradation of RNA by recognizing specific mutant RNAs.

TABLE 1. The Morbid Cutaneous Anatomy of the Human Genome

\begin{tabular}{|c|c|c|c|}
\hline Chromosome & Condition & Chromosome & Condition \\
\hline \multirow[t]{2}{*}{$1 \mathrm{p}$} & DNS & $11 q$ & Albinism \\
\hline & Ehlers-Danlos VI & & Tyrosinase - \\
\hline \multirow[t]{3}{*}{$2 q$} & Ehlers-Danlos (COL 3A1) & & Tyrosinase + \\
\hline & XP-B/CS & & Atopy \\
\hline & Waardenburg syndrome (HuP2) & $12 q$ & EBS (K5), EHK (K1) \\
\hline \multirow[t]{2}{*}{$3 p$} & Dystrophic EB & $13 \mathrm{q}$ & $\mathrm{XP}-\mathrm{G}$ \\
\hline & $\begin{array}{l}\text { Dominant (COL 7A1) } \\
\text { Recessive (COL 7A1) }\end{array}$ & $15 q$ & $\begin{array}{l}\text { Albinism } \\
\text { Tyrosinase + }\end{array}$ \\
\hline $4 q$ & Piebaldism (c-kit) & $16 p$ & Tuberous sclerosis \\
\hline $6 \mathrm{p}$ & BP Ag1 & $17 \mathrm{p}$ & SCC, BCC (p53) \\
\hline $9 p$ & Melanoma & $17 q$ & EBS (K14), EHK (K10), EHK-PPK (?K9), NF1 \\
\hline \multirow[t]{3}{*}{$9 q$} & $\mathrm{BCC}, \mathrm{KA}$ & & \\
\hline & Tuberous sclerosis & 18 & PV antigen \\
\hline & $\begin{array}{l}\text { XP-A } \\
\text { Nail-patella syndrome }\end{array}$ & $\begin{array}{l}19 q \\
X p\end{array}$ & XP-D \\
\hline $10 q$ & $\begin{array}{l}\text { BP Ag2 } \\
\text { BP }\end{array}$ & $\hat{X q}$ & $\begin{array}{l}\text { RXLI (sterold sultatase) } \\
\text { Ectodermal dysplasia }\end{array}$ \\
\hline
\end{tabular}

From Epstein EH Jr. The morbid anatomy of the human genome. Arch Dermatol 1993;129:1417-1423. 
A second mechanism would be to dilute the effect of the mutant gene by overexpression of the normal protein. A third mechanism would be to offer repair of the mutated gene. This might be done by transduction with a retroviral vector, direct in vivo introduction of DNA on various vectors such as gold particles bombarded into the skin, or topically applied DNA by transdermal delivery. Other potential mechanisms for gene therapy include grafting of normal stem cells and in vitro delivery of normal stem cells to the affected host.

Finally, 11 Cases of the Year were reported.

Dr. Ginat Larowski and colleagues (University of California at San Francisco) presented a case of iatrogenic scalding of a newborn in the nursery. This coincided with the elevation of water temperature that was instituted to combat an outbreak of Legionella. Careful monitoring of water temperature in the newborn nursery was emphasized. Seth Orlow suggested that this might represent a new form of the "staff"' scalded skin syndrome.

Dr. Julie Francis, (University of Washington) showed an unusual case of EB simplex in which the child had bullae and hyperkeratosis at birth, as well as neonatal scaling that resolved. Hyperkeratosis of the palms and soles was persistent. Histology showed lysis of the midspinous layer between what one would see in EB simplex (basal layer) and epidermolytic hyperkeratosis (subspinous layer). This disorder is probably a keratin abnormality. Discussion of whether it should be classified with the ichthyoses or bullous diseases ensued.

Dr. Steven Resnick (University of North Carolina), reported a patient with congenital erythropoietic porphyria that, because of its rarity, was misdiagnosed clinically as EB until the child was noted to have persistent, severe hemolytic anemia and photosensitive blistering. Decreased cosynthetase $3 \mathrm{ac}$ tivity confirmed the diagnosis.

Dr. Julie Prendiville (University of British Columbia) presented a patient with oral pemphigus vulgaris associated with inflammatory bowel disease and intercurrent herpes simplex virus gingivostomatitis. Review of the literature revealed that pemphigus vulgaris was associated with both of these entities in the past.

Dr. Yoke-Chin Giam of Singapore discussed a patient with nonfollicular Ofuji disease, which she called eosinophilic pustulosis. This child had abdominal lesions with individual sterile pustules coalescing into boggy plaques. Peripheral eosinophilia with a very high white blood cell count was also present.
Dr. Sylvia Suarez (Children's Memorial Hospital, Chicago) described an unusual ectopic meningomyelocele on the buttock but not in the midline. Dermatologists were warned that even nonmidline lesions in this area, especially of the depressed, atrophic type, may have connections to the spinal cord and associated spinal cord abnormalities.

Dr. Linda Beets-Shay (Alameda, California) presented a young girl with severe perioral and periorbital dermatitis that was granulomatous on biopsy. In addition, she had severe vulvar disease. She responded well to a combination of topical metronidazole and oral antibiotics.

Dr. Maki Goskowicz (San Diego, California) discussed an unusual case of Henoch-Schönlein purpura (HSP) with renal failure and severe pulmonary vasculitis. This complication, although rare, is significant because of $25 \%$ mortality. Less severe, subtle abnormalities in pulmonary function tests have also been reported in HSP.

Dr. Sheila Fallon-Friedlander (University of California at San Diego) described a 3-month-old patient with neonatal leukemia cutis in remission who developed tiny, scaly scalp papules that turned out to be leukemia cutis, heralding a relapse of the disease.

Dr. Howland Hartley (Children's National Health Center, Washington, D.C.) shared a fascinating case of a 3-year-old immunocompromised child with acute lymphatic leukemia who experienced a generalized, hyperkeratotic, erythematous, serpiginous, and annular eruption that on potassium hydroxide $(\mathrm{KOH})$ examination was teeming with Demodex. Elimite once daily every five days for three weeks cleared the condition. Dr. Hartley has subsequently seen four other children receiving chemotherapy for acute lymphoblastic leukemia with a similar disorder. Of note is the fact that, during monthly prednisone treatments, the skin disorder completely resolves.

Dr. Andrea Dominey (Baylor University) presented a child with a midline sternal defect and vascular anomaly with capillary hemangioma who improved only partially with steroid therapy and required tracheostomy for subglottic involvement. With the addition of $\alpha$-interferon, there was rapid (within $2 \mathrm{wks}$ ) $50 \%$ reduction in the size of the hemangioma, both internal and external. Although earlier reports stated that $\alpha$-interferon becomes effective only slowly, it is the experience of $\mathrm{Dr}$. Dominey and others in the audience that it can have a rapid and positive effect on massive hemangiomas. 


\section{Friday, July 30, 1993}

Dr. Maureen Rogers (Children's Hospital, Sydney, Australia) delivered the Sidney Hurwitz lecture entitled Hair Shaft Abnormalities: A Clinical and Microscopic Review. She discussed the structure of normal hair, and comprehensively reviewed hair shaft abnormalities in children.

Monilethrix is an autosomal dominant condition with variable penetrance and expressitivity. The hair is usually normal at birth, but is rapidly replaced within weeks or months by dry, dull, fragile hair. Spontaneous breakage leaves a stubble-like appearance. The scalp is usually affected, and eyebrows, eyelashes, and body hair may be involved as well. Follicular keratosis is commonly seen and may involve the scalp, nape of the neck, face, or limbs. Microscopic evaluation of the hair reveals elliptical or spindle-shaped nodes, separated by constricted internodes. Nodes have the diameter of normal hair, and may be medullated; internodes are narrower, usually nonmedullated, and are the sites of fractures. The beading may not be present in long hairs, and scraping of involved shorter hairs may be useful to obtain a diagnostic specimen. Node and intranode configurations have considerable variation, both within single hairs and from hair to hair in the same patient. Retinoids have been used with some success in some cases of monilethrix, although when treatment is stopped, hair is usually lost. Concern with toxicity and side effects limits their use as reasonable therapy.

Pseudomonilethrix was initially described as a developmental defect of hair, but has been shown to be an artifact caused by pressure-induced indentations in normal hair. Nodes appear wider than the normal shaft, with normal internodal thickness. This artifact is easily elicited by pressing overlapping hairs between glass slides, and is seen routinely when mounting the fair, fine hair of patients with certain ectodermal dysplasias. Similar deformities may be seen when handling the hair with fine-tooth forceps.

Pili torti is a rare anomaly characterized by twisting of the flattened hair shaft on its axis. The twisted hair is not actually narrowed, although it may appear so with casual observation. Examination of the light reflex on microscopy reveals the twist within the hair shaft. Classic pili torti is usually present at birth or in the first few years of life. It may be an isolated phenomenon or associated with other minor ectodermal abnormalities. Hair is spangled, dry, and brittle, and may break at different lengths. Bear-type pili torti is an autosomal dominant condition in which affected hair is coarse, stiff, and jet black. Eyebrow and lash hairs break off during childhood, with brittle hair and scalp alopecia generally noted after puberty. Pili torti may also be seen in a variety of other syndromes, including Bjornstad, Crandall, Menkes, arginosuccinicaciduria, and ectodermal dysplasias.

The pathogenesis of pili torti is known for Menkes syndrome. This $\mathrm{X}$-linked recessive disorder results from copper deficiency with impaired disulfide bond formation in hair keratin, as required enzymes are copper dependent. Hair is hypopigmented because tyrosinase is also a copper-dependent enzyme. Scalp hair is sparse and steely appearing, like tangled steel wool.

In trichorrhexis nodosa the hair microscopically appears fractured, with splaying and release of individual cortical cells from the main body of the shaft producing an appearance suggestive of the ends of two brushes pushed together. The term is appropriate for cases in which the defect occurs along the shaft, unlike trichoptilosis (split ends), in which the distal end of the hair may show fraying. Hairs are short and easily broken. Acquired trichorrhexis nodosa may be associated with physical injury to the hair shaft and has also been described in association with severe nutritional deficiency. The congenital disorder may be associated with other hair shaft abnormalities including monilethrix, trichothiodystrophy, woolly hair nevi, Netherton syndrome, and Menkes syndrome. Trichorrhexis nodosa is also associated with late-onset arginosuccinicaciduria.

Trichothiodystrophy is the term used to describe sulfur-deficient brittle hair, which is a marker for a neuroectodermal symptom complex. Scalp hair, eyebrows, and eyelashes are sparse and brittle, and break unevenly, giving a disordered appearance. Light microscopy reveals irregular outlines, flattened hair twists, and irregular angles. Polarizing microscopy reveals light and dark bands with and without birefringence.

Woolly hair is tight and curly, clearly different from that of other family members or other areas of the scalp. Autosomal dominant woolly hair has normal color and growth rate, with a decreased anagen phase. Autosomal recessive woolly hair is lighter and short. Associations with the disorder include keratosis pilaris atrophicans, palmoplantar keratoderma and endomyocardial dysplasia, cardio-faciocutaneous syndrome, and ocular abnormalities.

Pili annulati, or ringed hair, reveals alternating bright and dark bands when viewed with reflected 
light. This hair is not fragile, and its appearance is due to air-filled cavities within the cortex. It appears to be a defect of keratinization in the cortical cells.

Uncombable or spun glass hair classically is silvery blond, pale, and frizzy, and cannot be combed flat. Hair shaft anomalies such as longitudinal grooving and/or irregular cross sections are not specific for this entity.

Trichorrhexis invaginata is a hair shaft abnormality with a ball-and-socket configuration classically associated with Netherton syndrome. It is an autosomal recessive condition characterized by eczema, ichthyosis linearis circumflexa, the characteristic hair shaft abnormality, growth retardation, recurrent infections, hypernatremic dehydration, and elevated IgE levels. Studies suggest that trichorrhexis invaginata is due to a defect in hair keratinization.

The second speaker of the day, Dr. Vera Price (University of California at San Francisco), discussed clinical and research issues in alopecia areata. Alopecia areata is thought of as a reversible dysfunction of the hair follicle. It is a fairly common problem in pediatric dermatology, with half of the patients experiencing their first patch of hair loss before 20 years of age.

Dr. Price advocated different treatment strategies based on percentage of hair loss and age of the patient. Children 14 years of age or older, with less than $50 \%$ hair loss, may be given intralesional corticosteroids. Younger children generally will not tolerate this therapy. Instead, anthralin may be useful, beginning at a $0.5 \%$ concentration for 20 minutes, and advancing to 30-, 45-, and 60-minute applications. Anthralin concentration can be increased to $1 \%$ with a minimum of pruritus or erythema. Minoxidil 5\% solution is more effective than $3 \%$ solution; however, presently available $2 \%$ preparations (Rogaine) have limited efficacy. Combination therapy with minoxidil, anthralin, topical corticosteroids, and tretinoin has been used.

When hair loss is greater than $50 \%$, therapeutic options are limited. Wigs may be quite useful in improving patient self-esteem. Systemic corticosteroids are administered only rarely in children due to concerns about side effects, including cataracts. Other treatment modalities are topical immunotherapy, and the topical agents outline above. Psoralenplus ultraviolet A (PUVA) may also be used, but is not advised due to poor effectiveness and difficulties in administration. New therapeutic options for alopecia areata are restricted due to our limited understanding of its etiology. Families can benefit greatly from contact with the National Alopecia Areata Foundation.

Risk factors for a poor prognosis for alopecia areata include onset in childhood, duration greater than one year, extensive hair loss, history of atopy, other immune disease, or a positive family history.

The pathogenesis of this disorder is a reversible dysfunction of the hair follicle cycle. There is no obvious destruction of follicles in typical alopecia areata, and the potential for complete hair regrowth exists. Several possible target sites for the immune response and injury include cortical keratinocytes in the bulb region, cells of the dermal papilla, melanocytes, and stem cells in the bulge region. In a recent study done by Dr. Price and colleagues, no cell surface antibodies against autologous melanocytes were found in sera of patients with this disease.

Genetic factors may be important in the development of alopecia areata. Twenty percent of patients have a positive family history. Recent work by Dr. Price and colleagues looking at human leukocyte antisera (HLA) class 2 antigens in subtypes of alopecia areata was reviewed. Three groups of patients, all 18 years or older, were defined. Group 1 had alopecia areata of less than 6 months' duration, any extent; group 2 had alopecia of greater than 18 months' duration, with greater than $25 \%$ hair loss, and no history of alopecia totalis or universalis; and group 3 had alopecia totalis or universalis of greater than 2 years' duration. The data displayed an association of two well-defined clinical forms of alopecia areata (long-standing alopecia totalis/universalis and long-standing patch alopecia areata) with specific HLA antigens. The specific HLA 2 antigens may distinguish patients who are genetically predisposed to long-standing alopecia totalis/alopecia universalis. Dr. Price conjectured that susceptibility may be localized to distinct and limited nucleotide sequences in the allelic region.

The final speaker of the session, Dr. Paul Honig (Children's Hospital of Philadelphia), discussed head lice and tinea capitis. An estimated 3 to 12 million cases of head lice infestation occur per year, which incur an estimated cost of $\$ 357,000,000$, including patient care, lost parental wages, and school system expenses. Head lice should be considered in the diagnosis of any child who has pruritus, crusting, or excoriation of the posterior scalp. The differential diagnosis of nits includes seborrheic or atopic scales, hair casts, and artifacts (e.g., hair sprays). The distance of nits from the scalp surface may be useful in determining the presence of active infestation. Nits within 0.25 inch of the sur- 
face may represent active infestation, whereas those 0.50 inch or farther away are unlikely to be due to active lice.

Epidemiologic studies have shown that the vast majority of children with head lice have 10 or fewer lice; infestation with greater than 50 lice is quite uncommon. Lice are seen more commonly in Caucasians than blacks, with studies showing a relative ratio of 35:1. The female predominence has not been well explained. Lice do not survive at room temperature $\left(22^{\circ} \mathrm{C}\right)$ for more than 48 to 55 hours, and head-to-head infection is more common than fomite spread. Socioeconomic status does not appear to correlate with infestation; hygienic practices seem to be more important epidemiologically. Epidemics of head lice tend to occur in late summer and early fall, in contrast to those of pubic lice, which generally occur in winter months. This may be due to the temperature of scalp and inguinal areas, and the optimum 30 to $34^{\circ} \mathrm{C}$ temperature for hatching lice.

Dr. Honig stressed that treatment effectiveness is dependent on the quantity of medication used. Solutions or lotions are superior to shampoos or sprays, which may be diluted or kept on the hair for insufficient time. A new pyrethrin derivative, phenothrin, is a liquid shampoo that has been used in Europe and is quite effective for head lice, although it is not approved for human use in the United States.

Tinea capitis is most commonly caused by Trichophyton tonsurans in the United States. Trichophyton infections may cause scaling or dandruff, or, $40 \%$ of the time, inflammatory lesions, one-third of which are kerions. Kerions are considered to be due to an immune response rather than a bacterial infection. Children with kerions display delayed-type hypersensitivity reactions to Trichophyton antigen, unlike those without kerions. Dr. Honig reviewed his study of the bacteriology of kerions in 44 patients. Staphylococcus aureus was obtained from $50 \%$ of patients, either from surface cultures of the scalp or purulent material from the kerion, or both. Eight of 44 patients had gram-negative bacteria (Pseudomonas, Proteus, Klebsiella) cultured from the kerion or scalp. Four of them had moderate or heavy growth on semiquantitative testing.

It is uncertain if the bacteria represent contamination, colonization, or secondary infection. Further studies are necessary to define the potential role of pathogenic bacteria and the effect of treatment with antibiotics.

\section{Cases of the Year}

Dr. Linda Rabinowitz (Medical College of Wisconsin) presented a case of cutaneous mucinosis of infancy. A black girl was seen at 3 to 6 months of age with asymptomatic lesions of the trunk and upper extremities, which progressively increased in size and number over several years. Lesions were characterized as flesh-colored 2- to 3-mm, slightly firm papules that increased in size with coalescence. Pathologic evaluation revealed focal zones of loosely textured dermal stroma with wisps of basophilic material that were alcian blue positive at $\mathrm{pH}$ 2.5 , negative at $\mathrm{pH} 0.4$, and stained metachromatically with toluidine blue, consistent with mucin. Localized cutaneous mucinosis of infancy is characterized by numerous blue papules on the upper extremities and trunk. There are no systemic manifestations, although lesions are progressive. Dr. Rabinowitz reviewed two other forms of cutaneous mucinosis. Focal cutaneous mucinosis features solitary papules, without anatomic predilection, and without systemic disease. Surgical excision is curative. Juvenile cutaneous mucinosis results in widespread papules and plaques with periarticular nodules and joint inflammation. It is generally of acute onset, and improves spontaneously with time.

Dr. Kenneth Bloom (University of Minnesota) discussed an epidermic of $T$. tonsurans in a wrestling camp. An outbreak of inflammatory body ringworm occurred. Forty-three campers and one staff member had a presumptive diagnosis of tinea corporis, with $\mathrm{KOH}$ and culture proof in 25 individuals evaluated. All cultures obtained were positive for $T$. tonsurans. Epidemics of $T$. tonsurans infection have been reported both in camp and in health care settings.

Dr. Bloom also discussed monozygotic twins with hypomelanosis of Ito. The black infants were born at 32 weeks' gestation due to pregnancy complicated by chorioamnionitis. They had nearly identical feather-edged, hypopigmented patches extending across the midline in the abdominal area. Both infants had microcephaly, dolicocephaly, epicanthal folds, hypotonia, and mental and motor retardation. They were a product of a dichorionicdiamniotic placenta. Chromosomal analysis of the children's blood and skin revealed a normal karyotype with no evidence of chromosomal instability or mosaicism.

Dr. Rhonda Schnur (Children's Hospital of Philadelphia) discussed ectodermal abnormalities in the Johanson-Blizzard syndrome, which is character- 
ized by hypoplasia of the alae nasi, upswept patterning of the hair, and pancreatic insufficiency. A child was diagnosed with this syndrome at birth, with typical facial features, intrauterine growth retardation, failure to thrive, and normal pancreatic function. Cutaneous findings included translucent, pale, velvety skin with prominent vasculature, and scalp aplasia cutis. Cutaneous abnormalities not previously described with this syndrome were two clusters of port-wine stain on the midthoracic and lumbosacral area, dystrophic nails, and a congenital nevus. This child also had developmental delay and possible dental abnormalities. Dr. Schnur stressed that aplasia cutis is a cardinal feature of this syndrome.

Dr. Schnur also described a patient with hypertrichosis languinosa with ringed skin creases and facial dysmorphism. The child was noted at birth to have excessive hair, which has continually increased. The facies was abnormal, with bilateral epicanthal folds, a flat nasal bridge with upturned tip, and a cupid-bow mouth. The skin was characterized as redundant and doughy, with numerous folds without hyperelasticity. It is believed that this patient has a new variant of the hypertrichosis congenita syndromes.

Drs. Maira Alvarez-Franco and Amy Paller (Children's Memorial Hospital, Chicago) discussed a child and her family with generalized lentiginosis. A 6-year-old Hispanic girl had generalized hyperpigmentation; numerous lentigines of lips, palms, and soles; many café au lait spots; hyperpigmented spots; axillary freckling; microcephaly; and short stature. No Lisch nodules were present, and results of neurologic examination and computerized tomographic scan of the brain were normal. A 9-year-old brother had similar clinical features with the exception of microcephaly, and a 12-year-old sister had numerous hyperpigmented keratoses in the antecubital fossa, pathologically consistent with verrucous keratoses. A 25-year-old sister was well, but five or seven of the mother's siblings were affected. None of the affected family members had evidence of mental retardation, hearing or speech abnormalities, neurofibromas, or other tumors. Biopsy specimens from lentiginous lesions, as well as lesions that appeared to be typical café au lait spots, were diagnostic of lentigines. It was stressed that patients with generalized lentiginosis may have a variety of pigmentary changes, and that this entity should be considered in the differential diagnosis of neurofibromatosis type 6 .

\section{Saturday, July 31, 1993}

The final day of the meeting was devoted to pigment abnormalities and melanocytic nevi in children. Dr. Seth Orlow (New York University) discussed the genetic basis of hypopigmented diseases. He reviewed the major scientific breakthroughs that in the last decade have caused a revolution in understanding the genetics of hypopigmentation.

Part I included diseases in which melanocytes never arrive at their proper sites. Waardenburg syndrome is an autosomal dominant condition characterized by deafness, white forelock, and heterochromia irides. It has been studied with the aid of a murine model, the splotch mouse, leading to the discovery of an abnormal DNA-binding protein causing a subset of neural crest cells to differentiate abnormally. In piebaldism there is poliosis and depigmentation on the face and trunk. This condition may mimic vitiligo but is static and present at birth. Normally the c-kit proto-oncogene helps to determine melanocyte survival and migration, but in piebaldism this is abnormal. Vitiligo, an acquired condition that is not static, may be a delayed form of piebaldism. Again, a mouse model allowed gene mapping for vitiligo and revealed one allele at the locus that is also responsible for piebaldism.

Finally in this group, Dr. Orlow compared and contrasted nevus depigmentosus and hypomelanosis of Ito. Nevus depigmentosus is present at birth, usually segmental, and does not follow Blaschko lines. There is no lack of melanocytes, but a decreased rate of melanin production and abnormal transfer. Hypomelanosis of Ito may not be present at birth and follows Blaschko lines. There are decreased melanocyte numbers, decreased melanin production, and abnormal transfer.

Part II dealt with diseases in which melanocytes are present but do not produce melanin properly. Although tyrosinase is important in this complex process, it is not the only enzyme involved. Patients with tyrosinase-negative albinism (type $I$ ) cannot make pigment at all. The effects of ultraviolet light can be devastating. When screening these patients for skin cancer, it is important to remember that melanomas may appear pink (amelanotic). In type I albinism 40 to 50 different mutations have been found in the gene that encodes for tyrosinase, a copper-dependent enzyme critical for the production of melanin. Not all patients with tyrosinasenegative albinism have total lack of tyrosinase activity, as seen in yellow albinism (type IB). The hair bulb tyrosinase test can be helpful in determining 
tyrosinase activity, but it is very inaccurate in infancy and variable in adults. In one reported mutation, pigment appears only in acral (and thus cooler) body parts, illustrating the temperature-sensitive aspects of tyrosinase activity. This is best seen in the Siamese cat. Tyrosinase is not the only variable in albinism. In people with so-called brown albinism the defect appears to be a mutation that affects tyrosinase-related proteins I and II. These individuals have a light brown, café au lait color with photophobia and nystagmus. In another condition, Menkes syndrome, hypopigmentation is due to a copper transport abnormality that prevents normal tyrosinase activity. Of interest, carriers of this disease can have findings that mimic those of hypomelanosis of Ito.

The classic autosomal recessive form of albinism, formerly called tyrosinase-positive albinism, is now called type II oculocutaneous albinism. In the newborn it can be difficult to distinguish type I from type II. Dr. Orlow suggested three helpful findings in type II disease: retinal pigmentation, mongolian spots, and yellowish hair. The mutation for type II albinism has now been identified on chromosome 15q. This discovery, made using a mouse model, resulted from the observation that hypopigmentation is a feature of Angelman syndrome and Prader-Willi syndrome, both due to $15 \mathrm{q}$ abnormalities.

In part III, Dr. Orlow discussed diseases due to abnormal packaging and transport of melanin, specifically, three autosomal recessive conditions characterized by metallic or silvery hair. ChediakHigashi syndrome is associated with neurologic and immunologic deficits. The generalized hypopigmentation is due to abnormal transport of abnormal (giant) melanosomes. Griscelli syndrome may mimic Chediak-Higashi syndrome but is distinguished by a lack of Langerhans cells and inability to transport normal melanosomes. Elejalde disease is a static, nonprogressive neurologic condition, also with pigment dilution and silvery hair. All three conditions have in common an abnormality in lysosomederived organelles.

Finally, Dr. Orlow discussed HermananskyPudlak syndrome, an autosomal recessive disorder with a lysosomal abnormality that affects a variety of different organelles. These patients have hypopigmentation, reddish hair, and bruising. The bleeding disorder is due to the absence of platelet-dense granules with resultant aggregation abnormality.

In the second session of the day, Dr. Joseph G. Morelli (University of Colorado) lectured on viti- ligo, a condition caused by loss or destruction of melanocytes. He discussed the many theories of pathogenesis, the autoimmune theory being the most plausible. It is well known that the frequency of other diseases thought to be autoimmune is increased in patients with vitiligo and their families. Support for this theory also comes from studies demonstrating that patients' serum can kill cultured human melanocytes in vitro. However, antibodies may only be a marker for the process taking place. The other major theory concerns inherent defects in melanocytes. When harvested from patients, these cells are not normal. They contain autophagosomes, numerous cytoplasmic vesicles, and dilated, rough endoplasmic reticulum. This suggests a defect in formation or translocation of proteins. Dr. Morelli stated that vitiligo may be due to a number of different diseases with a common final result.

Vitiligo is not just a pigmentary disease. Keratinocytes show degenerative changes. There is increased expression of ICAM-1 and HLA-DR in the edges around depigmented skin, and decreased ICAM-1 in areas of depigmentation. Langerhans cells are also affected. Although normal in number, they have a functional impairment, with decreased sensitivity to contact allergens.

The clinical variability of vitiligo is striking. Trichrome vitiligo is more common (or more easily appreciated) in dark-skinned individuals. Inflammatory vitiligo, occurring in $5 \%$ of patients, has a red, raised, pruritic border. An intense inflammatory infiltrate is seen on biopsy specimens.

Vitiligo is a disease of childhood: in $25 \%$ of patients onset is before age 10 years, in $50 \%$ before age 20 . The prevalence is approximately $1 \%$ of the population, occurring equally in all races and with a slight female predominance. Segmental and generalized vitiligo may represent different diseases. Segmental disease is not dermatomal and seldom converts to the generalized type. Acral areas are often first affected in the generalized type, which tends to be symmetric and is often progressive. It evolves over a period of years, but seldom spreads rapidly. Spontaneous repigmentation is common.

No treatment for vitiligo is particularly effective, although PUVA is accepted as the best available, with 8-methoxypsoralen the preferred agent. $\mathrm{Pa}$ tients must be highly motivated to withstand the required year or more of twice-weekly treatments. Therapy is most likely to be successful in patients who already have perifollicular repigmentation. A poor response can be anticipated in segmental or acral disease. Topical steroids have been studied 
extensively in vitiligo, but in no carefully controlled studies. They require prolonged use, generally at least four months. Ehellin is not available in the United States. When combined with UVA phototherapy it has been successful in European countries but has a $30 \%$ frequency of hepatotoxicity. L-Phenylalanine has been used orally combined with UVA phototherapy, as an adjunct to PUVA, and as a $10 \%$ topical cream. Camouflage make-up is highly successful for limited areas of depigmentation. Sunscreens are a must for all patients with vitiligo to prevent skin cancer. They also aid in cosmesis by preventing the tanning of surrounding normal skin. Unproved treatment alternatives include melagenia (a placenta extact), Chinese herbal lotions and teas, Anapsos, and aspirin.

In the treatment of children, Dr. Morelli recommends starting with low-potency topical steroids for four to six months. If there is no response, the next step is oral L-phenylalanine with natural ultraviolet light. The starting dose is $500 \mathrm{mg} /$ day, increasing up to $50 \mathrm{mg} / \mathrm{kg} /$ day. The mechanism of action is unknown. Generally PUVA is not recommended before age 10 years. Surgical treatment with grafting is not appropriate or practical in childhood. Depigmentation with $20 \%$ monobenzone should be reserved for patients with greater than $50 \%$ surface area involvement. The frequency of spontaneous repigmentation in children makes this a poor choice in this age group.

In the final session of the meeting, Dr. David McLean (University of British Columbia) discussed melanocytic nevi in children, detailing results of the Vancouver mole study. The study was done in the Vancouver school system and included children of European-Caucasian (1146), Indo-Pakistani (68), and Asian (378) backgrounds. Moles were examined clinically, but not histopathologically, in a prospective study. One major question was, how many moles do children have? The average number per square meter in those age 6 to 7 years was 7.2 for girls and 8.8 for boys, increasing by age 18 to 17.6 and 17.7 , respectively. In general, skin color seems to be more important than race in predicting mole number per child. This is supported by examining the geometric mean number of moles per child in the three study groups: Asian and Indo-Pakistani children had similar counts that were at least 4 times less than in Europeans. In all groups, the number increased substantially with age. Similar trends were reported in an Australian study, but with substantially increased counts, probably reflecting the effect of ultraviolet light exposure.

Another major question asked by the study was, are factors associated with increased nevi in children also associated with increased melanoma risk in adults? Several published studies showed an increased melanoma risk associated with an increased number of nevi. At this time, this seems to be the single greatest risk factor.

The Vancouver study found no association between mole numbers and the following variables in Asian and Indo-Pakistani children: hair color, skin type, skin color, sunburn score, and tanning score. This was not the case in the European group, where the nevi per square meter were most increased in intermittently exposed skin. Hair color was not correlated with increased number of nevi when corrected for the other variables. Skin type as measured by parental recall of burning/tanning history was highly correlated with the total number of nevi. A tanning score created for the study demonstrated that ability to tan was inversely proportional to the number of nevi. Similarly, the sunburn score was directly correlated with mole number. Number of freckles was also strongly correlated. As expected, the number of moles on a child correlated with the number on his or her parents. This may reflect genetic factors, lifestyle, or both.

When data from this study were compared with those from a large study of patients with melanoma in western Canada, Dr. McLean's group found a site relationship between mole number and later melanoma. The torso in men and the legs in women were the sites with most melanomas. These sites had increased mole numbers in the. Vancouver study of children.

An important question remains. If the number of nevi in childhood correlates with a later risk of melanoma, can we intervene and reduce this risk? The key is to start early in a child's life. Dr. McLean is currently studying the effect of education about photoprotection and sunscreen use on the number of nevi. 
This document is a scanned copy of a printed document. No warranty is given about the accuracy of the copy. Users should refer to the original published version of the material. 